\title{
Development of MeV Ultrafast Electron Scattering Instruments at SLAC National Accelerator Laboratory
}

R. K. Li ${ }^{1}$, A. H. Reid ${ }^{1}$, S. P. Weathersby ${ }^{1}$, G. Brown ${ }^{1}$, M. Centurion ${ }^{2}$, T. Chase ${ }^{1}$, R. Coffee ${ }^{1}$, J. Corbett ${ }^{1}$, J. C. Frisch ${ }^{1}$, M. Guehr ${ }^{1}$, N. Hartmann ${ }^{1}$, C. Hast ${ }^{1}$, L. V. Ho ${ }^{1}$, K. R. Jobe ${ }^{1}$, E. N. Jongewaard ${ }^{1}$, J. R. Lewandowski $^{1}$, A. M. Lindenberg ${ }^{1}$, J. E. May ${ }^{1}$, D. McCormick ${ }^{1}$, X. Shen ${ }^{1}$, K. Sokolowski-Tinten ${ }^{3}$, T. Vecchione $^{1}$, J. Wu ${ }^{1}$, J. Yang ${ }^{2}$, H. A. Dürr ${ }^{1}$ and X. J. Wang ${ }^{1}$

1. SLAC National Accelerator Laboratory, 2575 Sand Hill Rd, Menlo Park, California 94025 USA

2. University of Nebraska-Lincoln, 855 N 16th Street, Lincoln, Nebraska 68588, USA

3. University of Duisburg-Essen, Lotharstraße 1, 47048 Duisburg, Germany

Recent years have witnessed rapid development of ultrafast electron and x-ray scattering instruments, aimed at enabling the studies of structural dynamics on the fundamental time and length scales $[1,2]$. Ultrafast electron diffraction (UED) and microscopy (UEM) using a few mega-electron volts (MeV) electron beams is a promising new $R \& D$ area which has the potential to open many opportunities for groundbreaking science [3-7].

Electrons and x-rays are complementary tools to study material structures. While x-rays primarily interact with electrons in matter, electrons are sensitive to both electrons and nuclei. Compared with hard x-ray, a few $\mathrm{MeV}$ electrons have $10^{5}$ times larger scattering cross sections, $10^{3}$ times shorter wavelength, and $10^{3}$ times less radiation damage per elastic scattering [8]. The strong interaction with matter makes electrons the ideal choice to capture information from nanoscale or smaller samples, particularly monolayer and gas phase samples. Electrons are also charged particles and can be easily focused by electromagnetic lenses to form high spatial resolution real-space images.

MeV UED and UEM can achieve much higher spatio-temporal resolution compared to their keV counterpart at 100-300 keV energies. State-of-the-art MeV electron sources have one order of magnitude higher field gradient, thus the generated electron beams have much higher brightness. Moreover, space charge effects, the essential limit to the machine performance, between the source and sample, are dramatically suppressed by $10^{2}-10^{3}$ times following the $\beta^{2} \gamma^{3}$ scaling, where $\gamma$ is the Lorentz factor and $\beta$ is the normalized speed.

To exploit the synergy and complementarity with the Linac Coherent Light Sources (LCLS), SLAC National Accelerator Laboratory recently launched a UED/UEM Initiative aiming at developing the world's leading ultrafast electron scattering instruments. A MeV UED setup has recently been built, commissioned, and brought into full operation at SLAC's Accelerator Structure Test Area (ASTA). The platform, as shown in Fig. 1, consists of a complete photocathode RF gun system, including a LCLStype S-band photocathode RF gun, a $5 \mathrm{~mJ}$ femtosecond Ti:Sapphire laser, the laser-RF synchronization system, a high power modulator and an S-band klystron. This system tremendously benefits from many common key technologies developed for LCLS. The setup is now serving an active ultrafast science program and supports R\&D on future UED/UEM instruments and accelerators.

The UED machine has already generated exciting science results: The machine provided very high SNR and stable intensity in the diffraction patterns, which enabled us to study the lattice dynamics and diffuse scattering of Bismuth polycrystalline samples at high precision. It sparked more interest in this 
extensively studied, but yet not fully understood system. As another example, we've observed ultrafast lattice dynamics in FePt nano-cluster thin films following laser excitation. The samples consist of 5-10 $\mathrm{nm}$ size FePt grains and are currently being developed for the next generation magnetic data storage media. The laser induced ultrafast electron and spin dynamics is currently being actively investigated using soft x-rays at the LCLS. While x-ray holography at LCLS will enable direct imaging of magnetic switching, the current UED and future UEM instruments open up complementary studies of the currently inaccessible lattice dynamics on comparable nanometer length scales.

The next milestone of machine develop will be performing gas phase MeV UED experiment. MeV electrons travel at a speed very close to that of the pump laser, thus the velocity mismatch issue, which is a major constrain for $\mathrm{keV} \mathrm{UED}$, is essentially eliminated with $\mathrm{MeV}$ beams. For example, for $100 \mathrm{keV}$ electrons, to keep the velocity mismatch below $100 \mathrm{fs}$ the gas target needs to be 40 um or thinner, while a few mm thick gas sample can be used for $2.5 \mathrm{MeV}$ electrons. We will also upgrade the machine repetition rate from $120 \mathrm{~Hz}$ to $360 \mathrm{~Hz}$ to minimize the data accumulation time.

There are several other exciting targets in the Roadmap of UED/UEM development: (1) to improve the temporal resolution from 100 fs to $<20$ fs level to study fast chemical processes; (2) to improve the probe size in diffraction mode from a few hundred $\mu \mathrm{m}$ to sub- $\mu \mathrm{m}$, which greatly eases the sample fabrication processes; (3) to develop single-shot UEM revealing real-space images with ps-nm spatiotemporal resolution.

\section{References:}

[1] M. Chergui and A. H. Zewail, ChemPhysChem 10, 28 (2009).

[2] R. J. D. Miller, Science 343, 1108 (2014).

[3] X. J. Wang, Z. Wu, and H. Ihee, in Proceedings of PAC03 (IEEE, Portland, OR, 2003), WOAC003.

[4] W. E. King et al., J. Appl. Phys. 97, 111101 (2005).

[5] P. Musumeci and R. K. Li, in ICFA BD Newsletter No. 59, ed by J. M. Byrd and W. Chou (2012).

[6] http://science.energy.gov/ /media/bes/pdf/reports/files/Future_of_Electron_Scattering.pdf

[7] H. Dürr and X. J. Wang eds. SLAC-R-1039 (2014).

[8] R. Henderson, Q. Rev. Biophys. 28, 171 (1995).
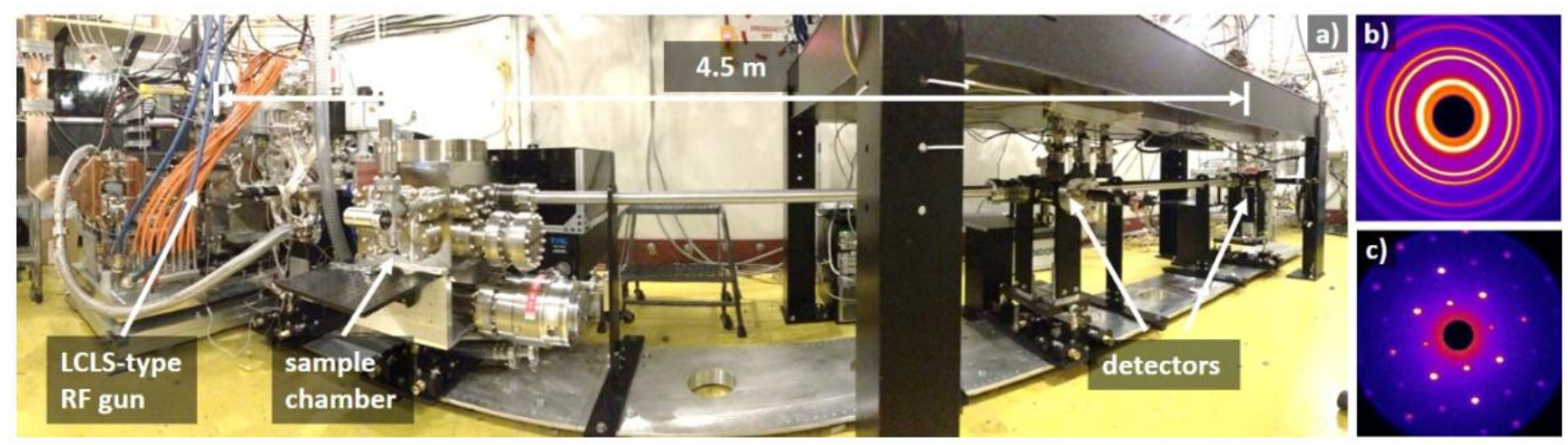

Figure 1. (a) The MeV UED beamline at SLAC's ASTA. Diffraction patterns of two recently studied system: (b) Bismuth and (c) FePt. 